\title{
Research on Simulation Calculation method and Energy-conserving analysis of Energy-Saving Conductors
}

\author{
Hongshan Zhang \\ Hebei Electric Power Design and Research Institute, Shijiazhuang, 050031, China
}

1178380809@qq.com

Keywords: Energy-saving conductors, resistance, calculation, effect analysis.

\begin{abstract}
Line losses are needed to be calculated before overhead transmission lines put into operation, and meanwhile AC resistance of wires determines its resistive losses and transmission capacity. In this paper, we compared several commonly used methods of AC resistance, obtain their advantages and disadvantages, and optimized choose the resistance calculation parameters. Finally, calculates the resistive losses of high conductivity steel core hard-drawn aluminum wire, aluminum alloy core aluminum wire and mid-strength aluminum alloy stranded wire in order to verify the accuracy of the algorithm, and provides a powerful data reference for the selection and design of wires in the future.
\end{abstract}

\section{Introduction}

All along, the overhead transmission line mainly adopts ACSR as a conductor. But with the continuous growth of the power load, the traditional transmission line transmission capacity has been unable to meet the rapid expansion of the demand, so the application of new energy-saving wire is imminent. Due to the construction of smart grid scale enlarging, in order to improve the conductivity, our country increase transmission capacity and reduce the transmission loss for target ${ }^{[1,2]}$.In order to develop new energy-saving wire such as steel core with high conductivity aluminum wire, aluminum cored aluminum stranded wire, aluminum alloy wire and other such strength wire . These new energy-saving wire with its excellent energy-saving effect has been applied to the new power transmission lines and the transformation of the old line ${ }^{[3]}$. Creating considerable economic benefits and social benefits, it has obvious popularization value ${ }^{[4-5]}$.

\section{Optimization calculation of AC resistance energy-saving wire}

The AC resistance of overhead transmission lines can determine the transmission capacity and resistance loss. With a wide extension of energy-saving wire in our country, the problem of quantitative analysis of new energy-saving AC resistance wire ${ }^{[6]}$ is inevi- table .The JCS-0374 algorithm and the Morgan formula are two kinds of common methods, but the results of these two methods are not exactly the same。 To select the AC resistance algorithm, this paper compared the Morgan algorithm, JCS-0374 algorithm and Bessel algorithm ${ }^{[7]}$ based on classical Maxwell equations, focused on the analysis of the three algorithms of the skin effect coefficient hysterics and eddy current loss coefficient, and analyzed the applicability of the method.

\subsection{Introduction to the algorithm}

(1)The algorithm based on Bessel

The skin resistance of conducts based on the Kelvin function is shown below:

$$
\frac{R}{R_{d}}=\frac{r_{0} \cdot \sqrt{\omega \mu \sigma}}{2} \frac{r_{0}^{2}-q_{0}^{2}}{r_{0}^{2}} \frac{K N-M L}{M^{2}+N^{2}}
$$

In type: $r_{0}$ and $q$ are the outer radius and inner radius $(\mathrm{m})$ of tubular cylindrical conductors respectively; $\delta$ is equivalent conductivity ( $/ \mathrm{M}$ )of conductor; $\omega=2 \pi f$ is the angular frequency (rad 
/ s); $\mu$ is the permeability $(\mathrm{H} / \mathrm{m}) . N, M, K$ 和 $L$ are skin effect coefficients of modified Bessel function calculation respectively.

(2)Morgan algorithm

In order to avoid the calculation of the Bessel function into the infinite series, the Morgan algorithm is more accurate and the approximate expression of the series is:

$$
\frac{\Delta R_{1}}{\Delta R_{d}}=Y_{s}(1-\Phi)^{-1 / 2}-1
$$

In style: $Y_{s}$ is the relative resistance increment caused by skin effect; $\Phi$ is the relative resistance increment caused by the proximity effect.

(3)JCS 0374 algorithm

On AC/DC resistance the JCS 0374 algorithm is considering the skin effect coefficient $k_{1}$ and core loss coefficients $k_{2}$, that is:

$$
K=\frac{R_{A C}}{R_{D C}}=k_{1} \cdot k_{2}
$$

\subsection{Comparison of the algorithms and analysis of sensitive factors}

(1) Coefficient of skin effect and proximity effect

Figure 1 plots the coefficient of proximity effect and skin effect between aluminum strand conductor and steel-cored aluminum strand conductor in the different working conditions.

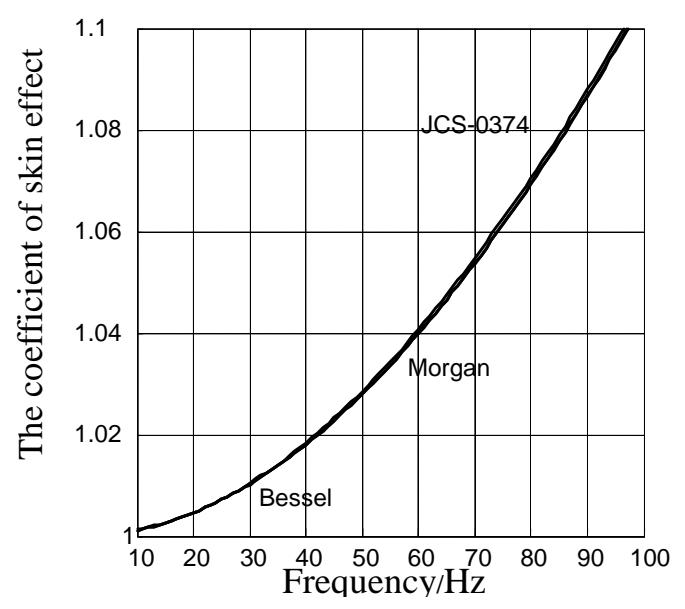

(a) Different frequency

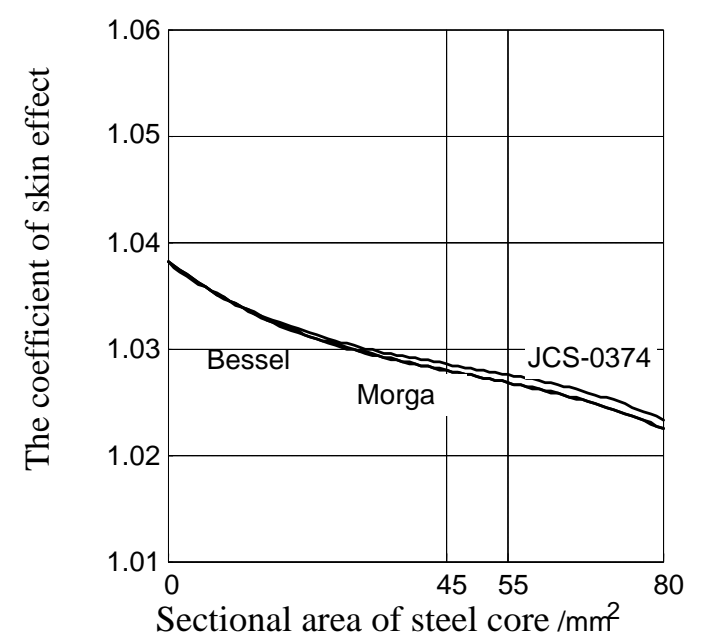

(c) Different sectional area of steel core

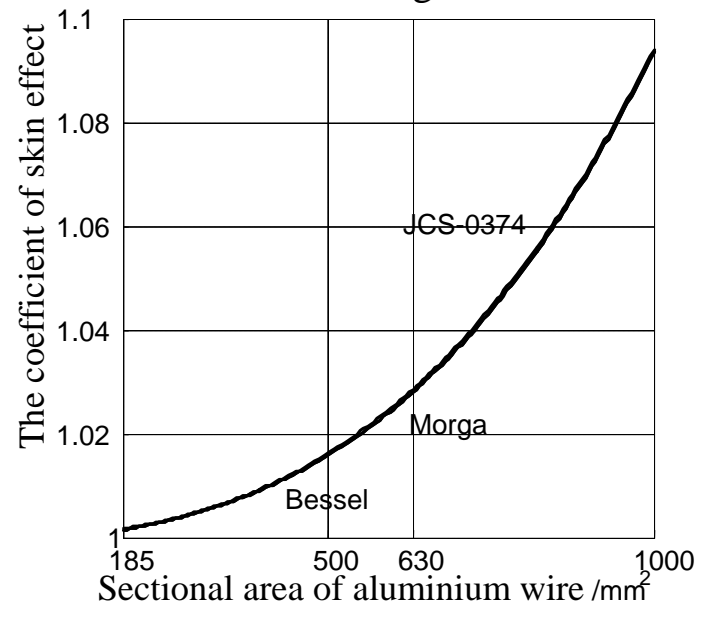

(b) Different sectional area of aluminium wire

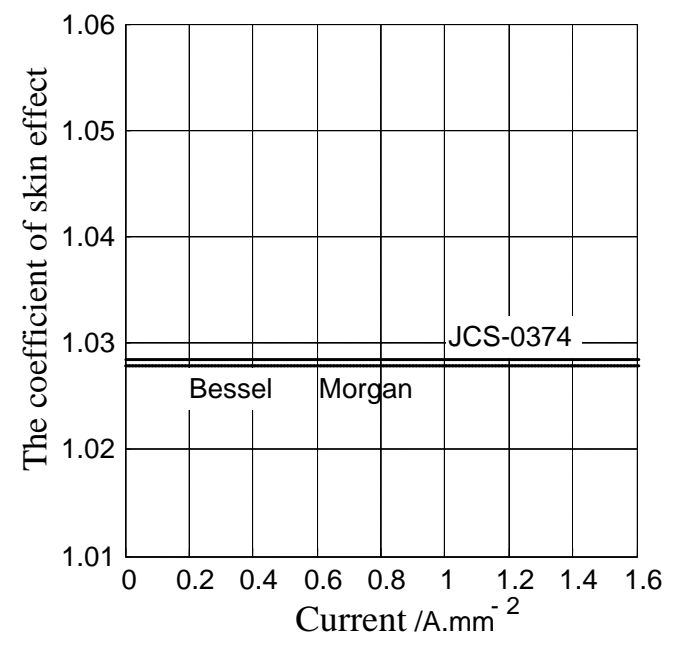

(d) Different current

Fig.1 Coefficient of skin-effect and proximity effect

From Figure 1 (a) and 1 (b), we can see that with increasing of working frequency or aluminum section, the skin effect the conductor becomes more and more obvious; When the aluminum section is 
unchanged, the skin effect of the wire decreases slightly due to the increasing of steel core section or the increasing of the DC resistance (see Figure 1 (c); and the current density almost does not affect the conductor skin effect (see Figure 1 (d).

Figure 2 plots hysterics and eddy current loss coefficients of ACSR in the different working time.
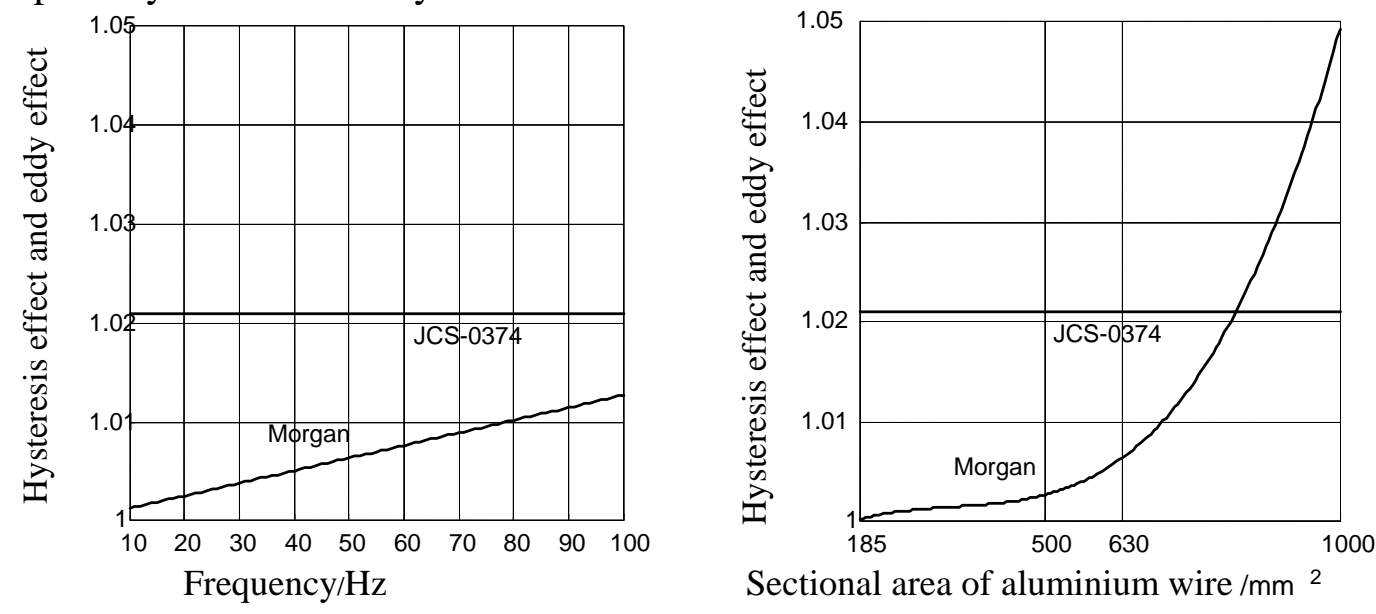

(a) Different frequency

(b) Different sectional area of aluminium wire
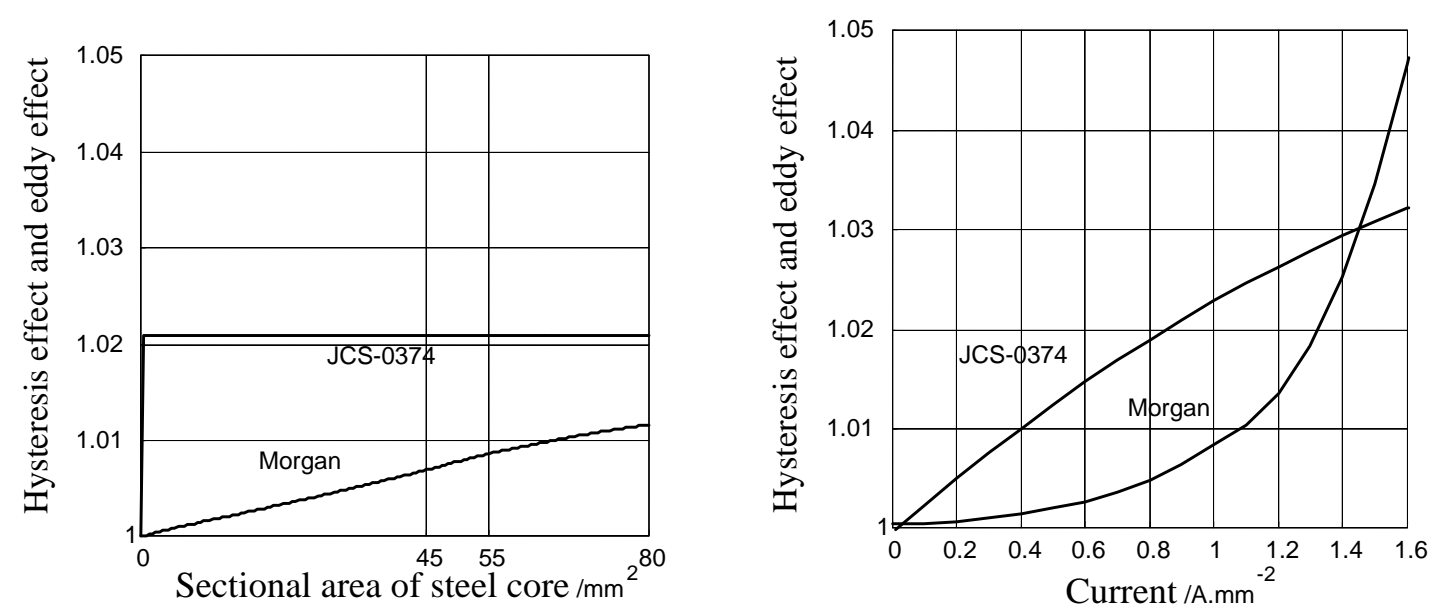

(c) Different sectional area of steel core

(d) Different current

Fig.2 Hysteresis effect and eddy effect

From the figure can be seen, steel core hysterics and eddy current loss with the increase of frequency, wire section and the current density. The growth rate is different. The theory of hysterics and eddy current loss also proved it.

\section{Optimization of parameter calculation of AC resistance}

\subsection{DC resistance}

In the 20 degrees Celsius considering the strand coefficient DC resistance of the single stranded wire ${ }^{[8]}$ is:

$$
R_{20}=\frac{4 \rho_{20} \lambda_{a m}}{\pi d^{2} N}
$$

The calculation of DC resistance under different temperature type is:

$$
R_{d}=R_{20}\left[1+\alpha\left(t_{c}-20\right)\right]
$$

\subsection{AC / DC resistance ratio}

The ratio of AC / DC resistance ratio is the ratio of the AC resistance and DC resistance when considering the skin proximity effect and the eddy current hysterics loss..

(1) the magnetic hysterics and eddy current loss of stranded wire

Resistance increment $\Delta R_{1}$ caused by eddy current and magnetic hysterics: 


$$
\Delta R_{1}=\frac{8 \pi^{2} a f\left(\sum_{1}^{m} N_{m}\right) \times 10^{-7}}{N^{2}} \times \mu \tan \delta
$$

Among them, $\mu \tan \delta$ is determined by magnetic properties of materials. If the current uniformly distribute in aluminum wires and ignore the axial current, the total magnetic intensity of ${ }^{[8]}$ wires can be calculated using the following equation:

$$
H=\frac{4 \pi l \sum_{1}^{m} N_{m}}{10 N} \times \frac{10^{3}}{4 \pi} \quad(\mathrm{A} / \mathrm{m})
$$

Values of $\mu \tan \delta$ are in the table 1 . Values of $\mu \tan \delta$ are related to the value of $H$ and the diameter of the wire. The value of $\mathrm{H}$ can be calculated accor-

ding to current I. If the value given by (10) formula is not equal to that given by Table 1 , the value $\mathrm{H}$ is ob- tained by interpolation method.

Tab.1 Value of $\mu \tan \delta$

\begin{tabular}{c|c|c|c|c|c}
\hline \multirow{2}{*}{$\begin{array}{c}\text { Diameter of wire / } \\
\text { mm }\end{array}$} & \multicolumn{5}{|c}{ Magnetic field intensity $H \times \frac{10^{3}}{4 \pi} /\left(A \cdot \mathrm{m}^{-1}\right)$} \\
\cline { 2 - 6 } & 0 & 5 & 10 & 20 & 30 \\
\hline $0.15 \sim 0.285$ & 1.00 & 7.13 & 35.84 & 345.6 & 267.2 \\
\hline $0.29 \sim 0.309$ & 1.15 & 10.8 & 46.20 & 326.7 & 247.2 \\
\hline $0.31 \sim 0.380$ & 1.30 & 14.4 & 56.55 & 307.8 & 227.2 \\
\hline
\end{tabular}

(2) The skin and the adjacent effect of the strand

Ignoring the conductivity of the steel core, the conductor can be regarded as a circular tube, the corresponding resistance increment for $\mathrm{R} 2$ is:

$$
\frac{\Delta R_{2}}{R_{d}}=Y_{s}(1-\varphi)^{-1 / 2}-1
$$

In type, $Y_{s}$ is the relative increment of resistance caused by the skin effect, $\varphi$ is the relative resistance increment caused by the proximity effect, and:

$$
Y_{s}=1+a(z)\left[1-\frac{\beta_{0}}{2}-\beta_{0}^{2} b(z)\right]
$$

In type:

$$
\begin{aligned}
& a(z)=\frac{7 z^{2}}{315+3 z^{2}} \quad(0<z<5) \quad, \quad b(z)=\frac{56}{211+z^{2}} \quad(0<z<5) \quad, \quad z=8 \pi^{2}\left[\frac{D-d_{s}}{2}\right]^{2} f r \quad, \quad \beta_{0}=\frac{D-d_{s}}{D}, \\
& \varphi=\lambda y^{2}\left[\frac{z^{2}\left(2-\beta_{0}\right)^{2}}{z^{2}\left(2-\beta_{0}\right)^{2}+16 \beta_{0}^{2}}\right], \quad y=\frac{D}{s}, r=\frac{1}{A R \times 10^{9}}, A=\pi \frac{D^{2}-d_{s}^{2}}{4}
\end{aligned}
$$

The AC / DC resistance $\beta$ is the ratio of AC resistance $R_{T}$ and DC resistance $R_{d}$ when considering skin and the proximity effect and eddy current and hysterics loss. That is:

$$
\beta=\frac{R_{T}}{R_{d}}=1+\frac{\Delta R_{1}}{R_{d}}+\frac{\Delta R_{2}}{R_{d}}
$$

\section{The calculation of examples}

A line uses $2 \times J L 4 / \mathrm{G} 1 \mathrm{~A}-400$ / 35.Its cross-sectional area is $426.2 \mathrm{~mm} 2$. In order to guarantee the comparability, the selection principle of wire sample is the same as the total cross-sectional area of the wire, so the cross-sectional area of the specimen is between $426.28 \mathrm{~mm} 2-426.2 \mathrm{~mm} 2$, which meets the calculation requirement.

\subsection{Wire selection}

The type of wires is shown in Table 2. 


\subsection{Calculation method}

According to the second chapter, use the Morgan method to calculate the DC resistance ratio.

\subsection{Calculation results of resistance loss}

The calculation results are shown in Table 3. In the line under the same information, the energy-saving effect of different types of wires is different. The steel core aluminum stranded conductor with high conductivity, aluminum alloy conductor with high conductivity and middle strength aluminum alloy conductor and so on show better energy saving effect and electrical characteristic than the ordinary steel core . Taking the steel core aluminum stranded conductor with high conductivity as an example, the electric energy loss of resistance per kilometer is also saved by about $0.71 \% \sim 2.77 \%$ than ordinary steel core aluminum conductor as the conductivity increased from $63 \%$ to $61.5 \%$.

\section{Conclusions}

(1) Considering all the factors, the results of Morgan algorithm is most close to that of Maxwell's classical electromagnetic theory, the applicable method.

(2) Energy-saving conductors such as steel core aluminum stranded conductor with high conductivity, aluminum alloy conductor with high conductivity and middle strength aluminum alloy conductor show the good energy saving effect, which has the alternative to the ordinary steel core aluminum wire application prospect and the energy saving effect of middle strength aluminum alloy wire is the best.

\section{References}

[1] HUANG Peng, MO Juan,WAN Jian-cheng,et al.Technical-economic analysis on application of energy-saving conductors in transmission lines [J].Electric Power,2013,46(7):153-157.

[2] ZHAO Li-yuan.Analysis on Economic Benefit of Energy-saving Wire in Transmission Line Engineering,Guangdong Science and Technology[J].2013,(20):130-131.

[3] WANG Guo-zhong.Discussion of a Simplified Calculation Method of AC Resistance of ACSR[J].Electric Wire and Cable,2010,(2):10-12.

[4] DING Guangxin,SUN Zhusen,ZHANG Qiang,et al.Analysis on Application of Energy-Saving Conductors in Transmission Lines[J].Power System Technology.2012,36(8):24-30.

[5] ZHANG Sixiang, HU Junpeng.Selection Research of Energy-saving Conductor of Transmission Lines[J].Shandong Electric Power, 2014,(1):20-25.

[6] ZHANG Ruiyong,ZHAO Xinyu,LI Ming et al.Polarization and Application of the New-type Energy-saving Conductors in Transmission Lines[J].Electric Power Construction, 2012: 32 (6) : 89-92.

[7] ZHANG R ui-yong,ZHAO Xin-yu,TAO Li-xue,Comparison of Calculation Methods of AC Resistance in Overhead Transmission Lines [J].Electric Wire and Cable,2014,(4):1-3.

[8] AC resistance calculation and analysis of Energy-saving[J].Low Carbon World,2013,(20):77-78.

[9] WAN Jian-cheng,LIU Long,FENG Liang et al.Improved Calculation of Loss Caused by Hysteresis and Eddy Current Effect Including Current Skin Effect[J].Electric Power,2014, 47 (1): 71-74. 